\title{
LA HISTORIA COLONIAL DE AGUASCALIENTES CONTADA POR BEATRIZ ROJAS
}

JESÚS GÓMEZ SERRANO

Departamento de Historia/UAA

BEATRIZ Rojas, Las instituciones de gobierno y la élite local. Aguascalientes del siglo XVII hasta la Independencia, México, El Colegio de Michoacán/Instituto Mora, 1998, 339 pp.

In 1979, algunos estudiantes de la primera generación de sociología de la UAA (1976-1981) nos empezamos a interesar en la historia local. Estimulados por la lectura de algunos textos del historiador francés Pierre Vilar, pero sin haber digerido del todo los libros de Agustín Cueva, Sergio de la Peña y otros teóricos del desarrollismo que querían explicar la historia de Latinoamérica con las herramientas proporcionadas por el análisis marxista, nos propusimos temas tan ambiciosos y a la vez tan inasibles como "la acumulación originaria de capital en Aguascalientes". Era evidente que la Historia de Aguascalientes de Agustín R. González, de la que todavía se encontraban en las librerías ejemplares de la pulcra edición hecha por Francisco Antúnez en 1974, iba en un sentido completamente distinto y de poco nos servía su lectura. Como nuestra formación historiográfica era bastante precaria, no entendíamos que el libro de don Agustín había sido escrito por un político en retiro, según los modelos vigentes en la segunda mitad del siglo XIX. Estábamos "ebrios de sintaxis y ciegos para la semántica" (Merton) y obviamente no encontramos en el libro de González los datos y elementos de análisis exigidos por nuestro paradigma teórico, En forma inevitable concluimos que la obra de González no servía para nada y que la historia de Aguascalientes estaba todavía por escribirse. En lo personal, tardé todavía algunos años en ser capaz de leer con provecho y de disfrutar ese texto, al que hoy en muchos sentidos estimo como un clásico de la historiografía regional mexicana. 
Aturdidos como estábamos por la lectura de esos teóricos, encontrábamos poco relevante y hasta carente de sentido la información que recogíamos en la sección histórica (por entonces en completo desorden) del Archivo General del Estado. ¿Qué gran proceso histórico ilustraba la venta de unas pocas fanegas de sembradura pertenecientes a la antigua hacienda de Pabellón? Al parecer, ninguno, o por lo menos ninguno que fuera relevante en el contexto de esa "gran historia" que imaginábamos.

\section{LA HISTORIADORA Y SU OBRA}

Entonces alguien descubrió un cuaderno de trabajo del INAH publicado en 1976, que se llamaba La destrucción de la hacienda en Aguascalientes, 1910-1931. Leerlo fue como refrescarse en un oasis después de una penosa travesía por un desierto lleno de espinosos conceptos. En ese trabajo, que en 1980 sería publicado como libro por El Colegio de Michoacán, encontramos lo que con tanto afán pero sin éxito buscábamos en los manuales marxistas: la significación de la historia menuda, el sentido y la importancia de algo que por entonces empezaba a llamarse "historia regional". A Beatriz Rojas, la autora de ese trabajo, le debemos la historia de la descomposición de la gran propiedad en la época de la Revolución, pero en un sentido más profundo y definitivo le debemos el primer acercamiento moderno a la historia regional. Sin que nos haya dado nunca una clase, gracias a su libro ejerció entre nosotros un verdadero magisterio.

Por lo que toca a sus trabajos sobre Aguascalientes, Beatriz abandonó la historia del periodo posrevolucionario y empezó a concentrar su atención en la época colonial. Poco a poco, con una paciencia y una constancia que vistas a la distancia no dejan de provocar admiración, empezó a ofrecernos los resultados de sus pesquisas. Primero publicó (1984) un artículo sobre la testamentaría del Pbro. Ignacio Rincón Gallardo y sus hermanas, que fue para la autora un primer acercamiento a la época en la que en lo sucesivo concentraría sus esfuerzos. Después (1986) nos dio a conocer su trabajo sobre "El cultivo de la vid y la fabricación de chinguirito", que es una penetrante radiografía de las estructuras de poder, las redes de influencia y la forma inextricable en la que se mezclaban los negocios y la política en la villa de Aguascalientes en el siglo XVIII. $\mathrm{Al}$ año siguiente publicó un artículo sobre el intento frustrado de los habitantes de Jesús María para fundar un segundo pueblo de indios en el sitio de Bocas. Hasta ese momento parecía que la autora 
iba para atrás y que terminaría escribiendo sobre la fundación de Aguascalientes o los chichimecas, pero en 1992 publicó un trabajo sobre la conformación y el funcionamiento del cabildo, y en 1994 otro más en el que se interesaba en las conflictivãs relaciones que mantuvieron Aguascalientes y Zacatecas a fines del siglo XVIII y principios del XIX. A estos artículos, que en conjunto constituyen una notable aportación a la cultura histórica regional, deben agregarse algunos otros de tono menor que se publicaron en el suplemento cultural El Unicornio.

Ahora que han sido reunidos en un solo volumen, ${ }^{1}$ podemos advertir con claridad que esos trabajos no son acercamientos dispersos a la historia colonial de Aguascalientes, sino aproximaciones sucesivas y llenas de intención a un tema y una problemática bien identificadas. Si Beatriz Rojas se hubiera detenido ahí, los aguascalentenses y en particular quienes nos interesamos profesionalmente en la historia de esta región tendríamos ya mucho que agradecerle. Sin embargo, todos esos artículos no fueron a la postre más que una especie de anuncio o preámbulo a la verdadera aportación de Beatriz a la historia colonial de Aguascalientes: su libro Las instituciones de gobierno y la élite local. Aguascalientes del siglo XVI hasta la Independencia, que apareció en la libre- rías a principios de este año bajo el sello editorial de El Colegio de Michoacán y el Instituto Mora. De muchas maneras, los artículos que publicó durante los últimos quince años están presentes en el libro, pero como cualquier lector podrá advertir, éste representa una aportación original y llena de sustancia. El conocimiento exhaustivo que tiene la autora de la época y los personajes, la amplia base documental en la que se apoya y ese dominio del oficio que le permite ofrecer una visión de conjunto convincente, vuelven este libro indispensable para cualquiera que se interese en el periodo colonial.

\section{LOS SEÑORES DE LA TIERRA}

La primera parte, titulada "Una sociedad y su relación con la tierra", estudia el problema crucial de la propiedad de la tierra: el proceso de formación de las grandes haciendas de la región, el carácter de sus dueños, la relación con los mercados mineros del norte y algo que, siguiendo a Eric van Young, llama la "ines-

1 Beatriz Rojas, En los caminos de la historia. Aguascalientes en el siglo XVIII, Aguascalientes, CIEMA, 1998. El volumen incluye dos artículos inéditos: "El padrón de 1792 en la subdelegación de Aguascalientes" y "Comercio y actividad económica en Aguascalientes: 1780-1810". 
tabilidad de la propiedad", es decir, la gran facilidad con que las haciendas cambiaban de dueño y alteraban sus límites. El impacto de las leyes castellanas que regulaban las herencias, la incertidumbre consustancial a los negocios ägrícolas, la estrechez de los mercados, el peso de las fundaciones piadosas y las terribles crisis que de manera periódica azotaban a la región son los factores que explican la tendencia de muchas haciendas a la subdivisión. Sin embargo, este proceso coexiste con otro de signo enteramente opuesto: la tendencia a la concentración de la propiedad. Acostumbrado a pensar en la hacienda como una institución sólida y estática, uno de los pilares del antiguo régimen, el lector descubrirá con sorpresa la forma tan sinuosa en la que se va escribiendo la historia de las haciendas de la región. En este sentido, un caso de estabilidad, tendencia a la acumulación y permanencia de los títulos en el seno de una misma familia a lo largo de varias generaciones, como el de las haciendas integradas al mayorazgo de Ciénega de Mata, viendo siendo algo así como la excepción que confirma la regla.

En el contexto de este análisis, es meritorio el esfuerzo que hace la autora por reconstruir a grandes rasgos la historia demográfica de la región. Apoyado en el trabajo inédito e inconcluso de Helio de
Jesús Velasco, Beatriz Rojas estima los volúmenes de población, su distribución por razas y el peso sorprendente que tenían los indios, que vivían no sólo en sus pueblos (San Marcos, San José de Gracia, Jesús María y San José de la Isla), sino dispersos en gran número en las haciendas y ranchos de la comarca. Aunque el tema exige un tratamiento más detallado y un mayor acopio de información (ojalá algún día Helio termine su trabajo), Beatriz logra demostrar en forma plausible que la población de la región no dejó de crecer a lo largo de toda la época colonial y que fue capaz de recuperarse de crisis tan terribles como la de 1785-1786. Este crecimiento constante de la población explica el dinamismo observado en el proceso de ocupación del suelo, la apertura de tierras al cultivo y el hecho de que, pese a tantas dificultades, nunca faltaron comerciantes o mineros dispuestos a hacer grandes desembolsos en la compra de las haciendas más ricas de la región.

\section{IMPORTANCIA DEL COMERCIO}

La segunda parte del libro está dedicada al comercio y a los comerciantes. Se trata de una actividad económica fundamental, ligada al surgimiento mismo de la villa en las postrimerías del siglo XVI, que le da a la región muchas de sus caracte- 
rísticas definitorias. Para un gran número de comerciantes, sobre todo durante el siglo XVII, la villa de Aguascalientes era tan sólo un punto en un amplio esquema de compra y distribución de mercancías que incluía la feria de Jalapa, los grandes almacenes de la ciudad de México y el poderoso imán de las minas del Norte. Con el paso del tiempo, la posición-comercial de la villa se consolidó y los mercaderes ricos empiezan a darle a la economía de la región un sello específico. Ya no se trata sólo del comercio, sino del arrendamiento de las alcabalas, el crédito a los hacendados y mineros, la inversión en bienes raíces y, en resumen, la participación de los comerciantes en la élite política y económica que decidía los asuntos de la región.

Beatriz hace algunas observaciones agudas sobre la actuación de los comerciantes peninsulares y los mecanismos que aseguraban su predominio. Inspirada en los hallazgos hechos por Brading en Guanajuato, ofrece el retrato de un inmigrante típico, que encuentra acomodo a la sombra de un tío, el cual es a su vez un miembro destacado de la oligarquía regional. Eso facilitaba la integración a la élite del recién llegado, abatía los costos del proceso de aprendizaje y le aseguraba un matrimonio ventajoso. De hecho, se observa el funcionamiento de mecanis- mos que aseguraban cierta perpetuación de la élite, fecundada por el arribo permanente de peninsulares

\section{LOS NEGOCIOS DE LOS ALCALDES}

En la tercera parte del libro, la autora estudia el funcionamiento del gobierno provincial. Lo primero que hace es preguntarse por la jurisdicción de la alcaldía que tenía su asiento en la villa de Aguascalientes; se trata de un territorio que se conformó gradualmente, "al compás del poblamiento del campo, de la mercedación de tierras y del establecimiento de las demarcaciones religiosas". En realidad, fue sólo a fines del siglo XVIII, cien años después de fundada la villa, cuando la alcaldía tuvo su configuración definitiva. En seguida, Beatriz se ocupa detalladamente de los alcaldes mayores, que fueron los funcionarios de mayor rango que hubo en la región. Después de señalar sus atribuciones, el origen de sus nombramientos, los mecanismos de remuneración y la forma en la que los alcaldes se integraban a la vida económica y social de la jurisdicción a su cargo, muestra con ejemplos concretos lo que implicaba en la época colonial el gobierno de una alcaldía.

Las relaciones entre la pequeña pero pretenciosa élite local y los alcaldes mayores no siempre eran buenas, como lo 
demuestra, entre otros, el caso de Nicolás Echeverría, que pagó más de 3 mil pesos por el cargo, pero que prefirió detenerse en Guadalajara, ciudad en la que, bajo la protección de sus poderosos amigos, se dedicó a jugar albures. La mala fama de este funcionario se extendió y de hecho el cabildo de Aguascalientes logró que su nombramiento fuera revocado. Lo sustituyó Agustín Ximénez de Muñana, un alcalde en cierta forma atípico, pues ejerció durante quince largos años el cargo (1757-1771), sin dar a los vecinos motivos de queja. De hecho, "en 1765 se le levantó un juicio de residencia y no se le encontró falta alguna".

Sin embargo, el curioso caso del alcalde Antonio Núñez de Toronjo tal vez demuestra que la falta de acusaciones no necesariamente remite a un expediente inmaculado. Pese a la gran cantidad de negocios que hizo, a los numerosos indicios de corrupción y a la forma descuidada en que ejerció el cargo, la gente no parece haberse quejado de él. Más escandaloso todavía fue el caso de Alejandro Vázquez de Mondragón, el último alcalde mayor que tuvo Aguascalientes. Gran aficionado a toda clase de juegos, permitía la lidia de toros en el pueblo de indios San Marcos "con el único fin de llenarse los bolsillos", pero lo más escandaloso es que terminadas esas fiestas se iba con sus toros al mineral de Asientos, donde los lidiaba de nuevo.

Ya desde entonces, la corrupción era no un accidente, sino el lubricante que permitía el funcionamiento de la maquinaría política gubernamental. La corrupción de los alcaldes, dice Beatriz Rojas, se aceptaba "como algo normal" y propio del cargo, que beneficiaba además a los vecinos con los que esos funcionarios se asociaban. A propósito de Eusebio Ruiz de Tejada, otro alcalde corrupto, la autora se pregunta si los vecinos de Aguascalientes encontraban tolerables los negocios sucios y los fraudes al fisco, pero no "las faltas a la moral", que fue lo que causó indignación en el caso de Nicolás Echeverría.

Con la promulgación de la Ordenanza de Intendentes, el lugar de los alcaldes mayores fue ocupado por los subdelegados de intendente. El primer funcionario que llegó con ese título a Aguascalientes fue Pedro de Herrera y Leyva, en abril de 1789; una de sus primeras y más importantes tareas fue oponerse a Felipe Cleere, el intendente de Zacatecas, quien por razones fiscales pretendía segregar la subdelegación de Aguascalientes de la intendencia de Guadalajara y agregarla a la suya.

Al final de cuentas, parece que el primer subdelegado fue el único con el que 
los vecinos de Aguascalientes lograron entenderse. De hecho, durante muchos años la subdelegación estuvo acéfala y el cabildo de la villa se erigió en los hechos en la máxima autoridad de la jurisdicción. Al preguntarse por las razones de este" prolongado vacío, Beatriz sugiere que los aguascalentenses se habían ganado fama de "quisquillosos" y su cabildo de beligerante, de tal manera que resultaba difícil encontrar al funcionario adecuado. De cualquier forma, lo prolongado de estas vacancias y los espacios abiertos al cabildo de la villa, remiten a las deficiencias de la administración colonial y a la necesaria politización de los cargos en el cabildo. De pasada, la subdelegación demostró que podía gobernarse sola y preparó el terreno en el que después de consumada la independencia nacional florecería el espíritu separatista de los aguascalentenses.

\section{BELIGERANCIA DEL AYUNTAMIENTO}

La cuarta y última parte del libro está dedicada al cabildo, el órgano de gobierno que incidía de manera más directa en los asuntos de la localidad. No sólo era la representación más visible del gobierno, sino que además tenía a su cargo las funciones de gobierno, justicia, hacienda y policía. Al regular el abasto de alimentos para la villa, administrar el agua para las huertas, vigilar los precios, normar las distracciones, dirimir conflictos entre particulares y contar con recursos para atender sus necesidades, el cabildo era sin lugar a dudas la institución más importante e influyente en la villa. Pertenecer al cabildo era muy significativo para los vecinos de la villa en términos simbólicos y por razones de prestigio, pero sobre todo por la posibilidad de hacer negocios, obtener prebendas y cuidar los intereses de la familia o el grupo de poder al que se pertenecía. El cabildo estaba en alguna medida sometido a la autoridad del alcalde mayor (a partir de 1789 del subdelegado) y sus atribuciones eran limitadas, lo que sin embargo nunca puso en entredicho su papel de gran regulador de la vida económica, política y social de la localidad.

Beatriz Rojas subraya que las actividades del cabildo pueden parecer intrascendentes y anodinas durante periodos dilatados, pero que en las situaciones de conflicto inmediatamente ocupa un primer plano. Al hablar de las últimas décadas del siglo XVIII, que caracteriza como conflictivas, hace notar que el cabildo es protagonista principal de todos los problemas que se vivieron: la formación de las milicias de la frontera de Colotlán, la prohibición de fabricar chinguirito, la gran 
crisis agrícola de 1785-86 y la integración de la subdelegación de Aguascalientes a la intendencia de Zacatecas, entre otros. La autora se pregunta por las razones de tanta intranquilidad y sugiere la conveniencia de encontrar en lo sucedido en otras alcaldías puntos de comparación. De cualquier manera, lo que no admite dudas es el protagonismo y la importancia del cabildo.

Todo ello sin olvidar que el cabildo funcionó también como un mecanismo de integración de la élite local. Con sorprendente regularidad los inmigrantes peninsulares codiciaban un cargo en el cabildo y pagaban lo que fuera necesario, pues al parecer no podían marginarse en una instancia de gobierno en la que se repartían prebendas y se dirimían conflictos. Lo que en cierta forma asombra es el buen entendimiento que prevaleció entre criollos y peninsulares en el seno del cabildo. Aunque hubo problemas y malos entendidos, derivados -dice con agudeza la autora- "del carácter altanero de los peninsulares y la sardónica maña de los criollos", unos y otros parecen haber actuado en el seno del cabildo sin el estigma o la impronta de su origen.

En resumen, el cabildo jugó a lo largo de toda la época colonial un papel central en la vida de Aguascalientes, y no sólo por sus atribuciones como órgano de go- bierno local, sino también como mecanismo de integración de la élite y espacio privilegiado para la solución de los problemas que aquejaron a la villa.

El tema de las relaciones entre el gobierno provincial y el gobierno local, ocupa la atención de un artículo muy reciente publicado por el joven historiador Francisco Javier Delgado. En plena coincidencia con lo dicho por Beatriz, Francisco Javier afirma que la Ordenanza de Intendentes no anuló las facultades ni el poder de los ayuntamientos, los cuales lograron mantener su preeminencia y su tradicional "libertad de acción". A pesar de sus atribuciones formales, los subdelegados se mantuvieron en una "posición de debilidad" y se enfrentaron no sólo con los ayuntamientos "sino sobre todo con las autoridades burocráticas establecidas por los reformadores borbónicos". ${ }^{2}$

\section{MAPAS E INDICES}

A lo largo de todo el libro, pero señaladamente cuando se ocupa del problema de la jurisdicción, Beatriz se ayuda en su exposición de una excelente serie de mapas,

2 "Subdelegados en Aguascalientes a fines del siglo XVIII. La aplicación de la Ordenanza de Intendentes", Caleidoscopio, núm. 5, enerojunio 1999, pp. 78-79. 
muy bien escogidos e inmejorablemente dibujados por el personal del departamento de publicaciones de El Colegio de Michoacán. Inspirada en los mapas de Phillip Powell y Peter Gerhard, la autora hace los suyos, que nos ayudan a los lectores a ubicar en el espacio los problemas planteados y desde luego los lugares de los que se hace mención. En total son diez los mapas que incluye el libro, pero en particular son de agradecerse el mapa de la subdelegación de Aguascalientes (1792), que se dibujó sobre un original del Archivo General de la Nación, y la magnífica reproducción facsimilar, en color, del Plano topográfico de la provincia de Zacatecas (1797), con todo y la leyenda en la que se defiende abiertamente la idea de incorporar el partido de Aguascalientes a esa provincia. Igualmente plausible es la preparación de un índice onomástico y otro toponímico, que facilitan la consulta del libro.

\section{DOS OBSERVACIONES CRÍTICAS}

Sin tratar de opacar los grandes méritos de la obra, quiero hacer dos observaciones de carácter crítico. La primera se refiere a la forma de citar de la autora, irrelevante para un lector común, pero muy importante para los investigadores, pues nos priva de eso que llaman el "be- neficio de la duda", que no es otra cosa que la oportunidad de hacer nuestras propias pesquisas sobre los temas cuyo interés compartimos. Con cierta frecuencia Beatriz no precisa el fondo documental del que tomó una referencia, o no indica el nombre del escribano, o proporciona sólo la fecha de una escritura, o se remite a fondos documentales que, como tales, en realidad no existen. Por experiencia propia sabemos lo engorroso que puede ser a veces dar una cita precisa, pero se trata de una de esas tareas ingratas a las que, por honestidad intelectual y respeto a nuestros lectores, estamos obligados los autores.

La segunda observación tiene que ver con cierto uso que hace Beatriz de la bibliografía relacionada con los temas que le interesan. Aplaudo la recurrencia constante a los trabajos clásicos de François Chevalier, Peter Bakewell, David Brading, Phillip Powell, Ramón Ma. Serrera, Richard Garner, Frederique Lange y Thomas Calvo, de los que Beatriz toma aliento analítico e información concreta, pero lamento que no haya hecho otro tanto con la bibliografía referida más directamente a la región de Aguascalientes. Como ella misma señala, esa bibliografía es escasa y tal vez en su conjunto se pueda tachar de humilde, pero justamente por esta razón se antojaba necesaria su 
revisión detallada. La primera y tal vez más grave omisión que encuentro es la de los trabajos del profesor Alejandro Topete del Valle, cuya Guía para visitar la ciudad y el estado de Aguascalientes se incluye en la bibliografía y es citada una vez, pese a lo cual no parece haber sido objeto de una revisión provechosa. Todos sabemos que la forma poco metódica en la que trabajó el profesor Topete dificulta la consulta de sus libros y artículos, pero creo que Beatriz tuvo tiempo más que suficiente para revisar su monografía Estampas de Aguascalientes y sobre todo los dieciséis números del Boletín de la Sociedad de Historia, Geografía y Estadística de Aguascalientes, que publicó don Alejandro entre 1934 y 1935. Como bien sabemos todos los que lo hemos revisado, en el Boletín hay una gran cantidad de documentos -transcritos con pulcritud y comentados con atingencia- sobre la historia colonial de nuestra región.

Beatriz tampoco parece haber consultado las Noticias histórico-jurldicas sobre la fundación de Aguascalientes, de Jesús Antonio de la Torre, ni las Noticias históricas sobre Aguascalientes contenidas en los libros de gobierno de la audiencia de Nueva Galicia, 1700-1710, de Carlos Aguirre. Este último es un opúsculo sin pretensiones analíticas, que no obstante es apreciable por la cantidad de informa- ción que reúne y ordena. En este caso la omisión puede disculparse si se atiende a la forma tan limitada en que circuló la edición. El Bosquejo histórico de Zacatecas, de Elías Amador, también tendría que haber sido objeto de una revisión cuidadosa; Beatriz lo incluye en su bibliografía y lo cita una vez, pero le cambia el título y no indica que edición consultó, lo que me hace pensar que no fue leído con atención. Con el manuscrito inédito de Helio de Jesús Velasco (Desarrollo demográfico de Aguascalientes, 1620-1820) comete una pequeña injusticia, pues se vale muy provechosamente de él y, aunque da los correspondientes créditos al pie de página, lo omite en la bibliografía.

Un caso curioso es el del libro La insurgencia en Aguascalientes, de Vicente Ribes, publicado por la UAA en 1989. Beatriz lo incluye en su bibliografía, pero me parece que no lo leyó, pues de otra manera no se puede explicar que no lo confronte cuando habla de la formación de las milicias de Colotlán, de la integración de los peninsulares a la élite regional, de los desacuerdos entre el cabildo de la villa y las autoridades provinciales y de los excesos del alcalde mayor Alejandro Vázquez de Mondragón, temas todos ellos a los que Ribes se refiere detalladamente en el capítulo II de su libro. No sé si Beatriz lo haya advertido, pero 
Vicente la precedió en la consulta de los documentos del Archivo General de Indias y del Archivo General de Simancas (ella lo llama Histórico) que dan soporte al análisis, lo que en mi concepto ameritaba por lo menos una referencia de corteš́a, si no es que un ejercicio de sano contraste entre sus conclusiones y las del historiador valenciano, cuyo segundo apellido, por cierto, es Iborra y no Iborres, como se asienta en la bibliografía.

Por lo que toca a mis trabajos, Beatriz hace en la introducción una amable referencia, que desde luego agradezco. Sin embargo, me parece que no los revisó o que por alguna otra razón prefirió ignorarlos en el desarrollo de su exposición, pese a que, como ella misma dice, en ellos hay muchas referencias a temas de historia colonial. Es revelador, por ejemplo, que uno de los dos libros a los que alude en la introducción no esté incluido en la bibliografía. Ciertamente, las pesquisas que hizo en los archivos le permiten referirse en forma directa a los temas, problemas y personajes cuyo interés compartimos, pero hay puntos de discusión que hubiera sido bueno hacer explícitos. Pienso en particular en sus reflexiones sobre los problemas que se suscitaron entre Zacatecas y Aguascalientes a fines del siglo XVIII, que en forma muy obvia se cruzan con los argumentos que defiendo en mi libro La creación del estado de Aguascalientes, publicado por el CoNACULTA desde 1995. No sé si en Beatriz haya un prurito de originalidad, pero me parece que siempre es sano estudiar los trabajos de los colegas y partir en los propios de las aportaciones hechas por ellos, lo que no excluye de ninguna manera la discusión abierta e incluso el señalamiento de los puntos débiles que creemos advertir.

Estas consideraciones no tratan de ninguna manera de regatearle méritos al trabajo de Beatriz Rojas. Me atrevo a hacerlos porque creo que la crítica abierta, mesurada y objetiva es un aspecto de la tarea a la que estamos obligados los historiadores con respecto al trabajo de los colegas. Pensando en términos generales o en un tema o una región particulares, es claro que el conocimiento histórico avanza normalmente gracias a la coincidencia $-\mathrm{y}$ a las diferencias, que muchas veces resultan más estimulantes que aquellasde esfuerzos de carácter individual. El primer deber de un historiador consiste en hacer aportaciones originales y sustantivas al conocimiento de la época, la región o la problemática que estudia, pero este deber trae aparejada la obligación de seguir con atención, generosidad y espíritu crítico los esfuerzos de los colegas. Pocos espectáculos más tristes en nues- 
tras universidades e institutos de investigación que ese silencio deliberado que tantas veces acompaña la aparición de libros llenos de información nueva y de planteamientos sugestivos.

\section{UN LIBRO FUNDAMENTAL}

Las instituciones de gobierno y la élite local ofrece una penetrante y sugestiva visión de conjunto sobre la época colonial en Aguascalientes. Con él, Beatriz Rojas culmina un esfuerzo que inició hace casi veinte años y del que conocimos sus primeros frutos en 1984. Puede sentirse satisfecha, pues le vio el fin a una prolongada jornada y se liberó de "un fardo", como escribió en la dedicatoria de mi ejemplar. Dueña del oficio y de su tema, Beatriz Rojas ha consumado con este libro una aportación fundamental a la historiografía regional de Aguascalientes. 